Gut and Liver, Vol. 10, No. 1, January 2016, pp. 42-50

\title{
Therapeutic Decision-Making Using Endoscopic Ultrasonography in Endoscopic Treatment of Early Gastric Cancer
}

\author{
Jong Yeul Lee, II Ju Choi, Chan Gyoo Kim, Soo-Jeong Cho, Myeong-Cherl Kook, Keun Won Ryu, and Young-Woo Kim \\ Center for Gastric Cancer, National Cancer Center, Goyang, Korea
}

\section{See editorial on page 3.}

Background/Aims: We evaluated the effectiveness of an endoscopic ultrasonography (EUS)-based treatment plan compared to an endoscopy-based treatment plan in selecting candidates with early gastric cancer (EGC) for endoscopic submucosal dissection based on the prediction of invasion depth. Methods: We reviewed 393 EGCs with differentiated histology from 380 patients who underwent EUS from July 2007 to April 2010. The effectiveness of the EUS-based and endoscopy-based plans was evaluated using a simplified hypothetical treatment algorithm. Results: The numbers of endoscopically determined mucosal, indeterminate, and submucosal cancers were 253 (64.4\%), 56 (14.2\%), and 84 (21.4\%), respectively. Overall, the appropriate treatment selection rates were $75.3 \%$ (296/393) in the endoscopybased plan and $71.5 \%(281 / 393)$ in the EUS-based plan $(p=0.184)$. For endoscopic mucosal cancers, the appropriate treatment selection rates in the endoscopy-based plan were 88.1\% (223/253), while the use of an EUS-based plan significantly decreased this rate to $81.4 \%(206 / 253)(p=0.036)$. For endoscopic submucosal cancers, the appropriate selection rates did not differ between the endoscopy-based plan $(46.4 \%, 39 / 84)$ and the EUS-based plan (53.6\%, 45/84) $(p=0.070)$. Conclusions: EUS did not increase the likelihood of selecting the appropriate treatment in differentiated-type EGC. Therefore, EUS may not be necessary before treating differentiated-type EGC, especially in endoscopically presumed mucosal cancers. (Gut Liver 2016;10:42-50)

Key Words: Early gastric cancer; Conventional endoscopy; Endosonography; Endoscopic submucosal dissection

\section{INTRODUCTION}

Endoscopic submucosal dissection (ESD) has been widely employed as a treatment option for differentiated early gastric cancer (EGC) with minimal risk of lymph node metastases because it is less invasive and offers a better quality of life than surgical resection. ${ }^{1,2}$ Therefore, the proper selection of candidates for ESD is important in order to avoid both unnecessary surgery and the need for retreatment after ESD. Accurate preoperative prediction of invasion depth in EGC is essential for the selection of patients for ESD.

Conventional endoscopy has been a useful diagnostic modality for evaluating invasion depth of EGC. Previous studies have demonstrated that conventional endoscopy has an accuracy of $72 \%$ to $84 \%$ for evaluating depth of invasion (mucosa vs submucosa) in EGC..$^{3-5}$ Endoscopic ultrasonography (EUS) has been used for the locoregional staging of gastric cancer and is regarded as the best available method for the assessment of invasion depth. ${ }^{6-8}$ However, the role of EUS in distinguishing mucosal from submucosal cancer in EGC has not been established. Recent studies with large number of patients have reported accuracies of $67 \%$ to $82 \%$ for staging invasion depth (mucosa vs submucosa) of EGC using EUS. ${ }^{5,9,10}$ Reports comparing the accuracy of EUS with that of conventional endoscopy have produced inconsistent results. ${ }^{5,10-12}$ Yanai et al., ${ }^{12}$ for example, reported that EUS was useful in combination with conventional endoscopy for evaluating depth of invasion of EGC. Other studies, however, found that EUS failed to improve the accuracy of EGC invasion depth assessment, compared to the accuracy achieved with conventional endoscopy alone; this suggests that EUS may not be routinely necessary., ${ }^{5,10,11}$ Therefore, the role of EUS for the accurate preoperative prediction of invasion depth in EGC remains controversial. Furthermore, few studies have examined the impact on EUS-based clinical decision-making to

Correspondence to: Il Ju Choi

Center for Gastric Cancer, National Cancer Center, 323 Ilsan-ro, Ilsandong-gu, Goyang 10408, Korea

Tel: +82-31-920-2282, Fax: +82-31-920-1127, E-mail: cij1224@ncc.re.kr

Received on October 15, 2014. Revised on December 23, 2014. Accepted on December 31, 2014. Published online June 19, 2015

pISSN 1976-2283 eISSN 2005-1212 http://dx.doi.org/10.5009/gnl14401

@) This is an Open Access article distributed under the terms of the Creative Commons Attribution Non-Commercial License (http://creativecommons.org/licenses/by-nc/4.0) which permits unrestricted non-commercial use, distribution, and reproduction in any medium, provided the original work is properly cited. 
the preoperative evaluation of EGC. Thus, we conducted a study to evaluate the effectiveness of EUS-based treatment plan compared with endoscopy-based treatment plan for selecting appropriate candidates for ESD focused on the prediction of invasion depth.

\section{MATERIALS AND METHODS}

\section{Patients}

From July 2007 to April 2010, data of conventional endoscopy and EUS regarding 779 lesions in 756 patients with an endoscopic diagnosis of EGC were prospectively collected at the National Cancer Center, Goyang, Korea. Of the 779 EGCs, 296 lesions were excluded from analysis because of undifferentiated or mixed histology from histologic evaluation of the endoscopic biopsy specimens, and 90 lesions were excluded because of undifferentiated or mixed histology from histologic evaluation of the resected specimens. Thus, a total of 393 lesions in 380 patients was included in this retrospective analysis. The Institutional Review Board of the National Cancer Center approved this study (NCC2014-0024).

\section{Endoscopic invasion depth assessment}

Conventional endoscopic examination was performed by four expert endoscopists (I.J.C., C.G.K., J.Y.L., S.J.C.) using a conventional white-light endoscope (GIF-H260; Olympus, Tokyo, Japan). Indigocarmine solution was sprayed on the lesion and surrounding mucosa in order to enhance the surface details. Macroscopic type of gastric cancers was classified using the Paris endoscopic classification of superficial neoplastic lesions: ${ }^{13}$ type I (protruded), type IIa (superficial, elevated), type IIb (flat), type IIc (superficial, depressed), and type III (excavated). Tumor location was described by the area of the stomach involved: upper, middle, and lower. Ulceration was defined as a lesion with ulceration or fibrous scar. Endoscopic invasion depth assessment was classified as follows (Fig. 1): mucosal cancer (protruding lesions with a smooth surface, lesions with a shallow and smooth-surfaced depression, or flat lesions), and submucosal cancer (lesions that showed a more uneven stiffened base with an irregularly shaped nodule, or lesions with folds that were interrupted and enlarged). ${ }^{3,12}$ Lesions that could not be classified as either mucosal or submucosal cancers because of ambiguous morphology were designated as indeterminate cancers.
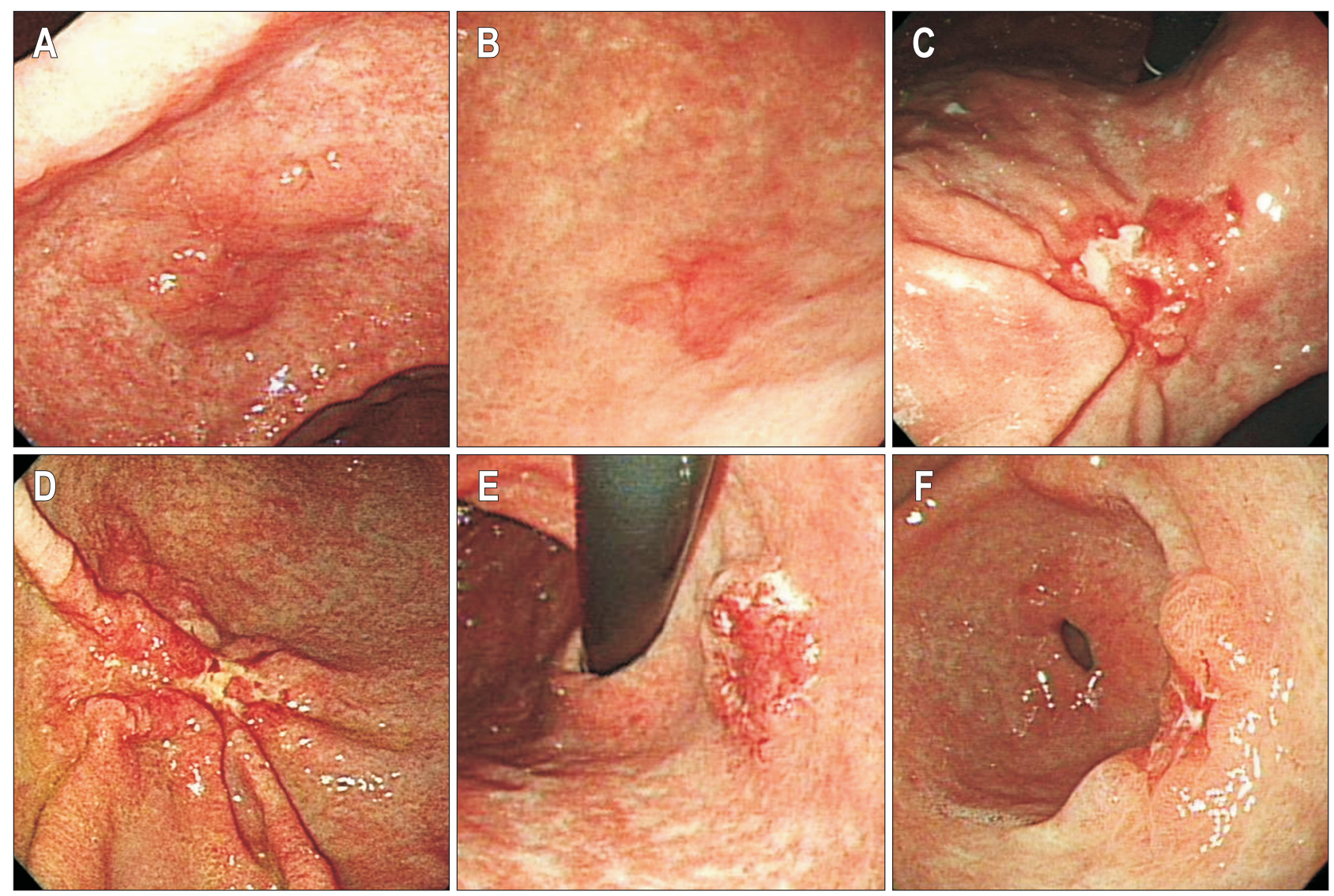

Fig. 1. Endoscopic assessment of the depth of invasion in early gastric cancers. Protruding lesions with a smooth surface (A), as well as shallow, depressed, or flat lesions with a smooth surface (B) were considered mucosal cancers. Lesions with an uneven, stiffened base and irregularly shaped nodules or those with enlarged or fused folds (C, D) were considered submucosal cancers. Lesions with ambiguous morphologies were classified as indeterminate cancers (E, F). 


\section{EUS invasion depth assessment}

EUS was performed under conscious sedation using midazolam (2.0 to $7.0 \mathrm{mg}$ ) within 2 weeks after endoscopy. EUS was performed using a radial echoendoscope (GF-UM2000; Olympus). On EUS, the first and the second layers correspond to the mucosa, the third layer to the submucosa, the fourth layer to the muscularis propria, and the fifth layer to the serosa. ${ }^{14,15}$ Assessment of invasion depth using EUS was rated according to the layer of tumor invasion: mucosa-submucosa1 (m-sm1) (tumor invasion to the first and/or second layer but not to the third layer), submucosa2 (sm2) (tumor invasion to the third layer but not to the fourth layer), and proper muscle or beyond (tumor invasion beyond the third layer). We considered lesions with tumor invasion that were confined within the second layer on EUS to be mucosal or submucosal 1 cancers because cancers with minute submucosal invasion $(<500 \mu \mathrm{m}$ from the muscularis mucosae) were very difficult to differentiate from purely mucosal lesions on EUS. For lesions with ulceration, those with smooth tapering of the submucosal layer were considered to be mucosal cancers, those with irregularity of the submucosal layer were classified as submucosal cancers, and those with abrupt interruption of the tapered submucosal layer were considered to be cancers invading the proper muscle or beyond. ${ }^{16}$

\section{Histologic evaluation}

Endoscopically or surgically resected specimens were fixed in 10\% formalin. Specimens resected endoscopically were sectioned in 2-mm intervals, and those resected surgically were sectioned in 4-mm intervals. Sectioned specimens were evaluated pathologically according to the World Health Organization classification of gastric cancer. ${ }^{17}$ Well- to moderately-differentiated adenocarcinoma and papillary adenocarcinoma were defined as differentiated type adenocarcinomas, whereas poorlydifferentiated adenocarcinoma, signet ring cell carcinoma, and mucinous adenocarcinoma were defined as undifferentiated type adenocarcinomas. The depth of submucosal invasion in resected specimens was classified into two groups using the Japanese classification of gastric carcinoma: ${ }^{18} \mathrm{sm} 1$ (invasion into the submucosa $<500 \mu \mathrm{m}$ from the muscularis mucosae) and sm2 (invasion into the submucosa $\geq 500 \mu \mathrm{m}$ from the muscularis mucosae).

\section{Endoscopy-based and EUS-based treatment plan}

To evaluate the effectiveness of EUS-based treatment plan compared with endoscopy-based treatment plan for selecting the appropriate treatment method, we used a simplified hypothetical treatment algorithm focused on the depth of invasion by conventional endoscopy and EUS. Endoscopy-based treatment plan was as follows: mucosal or indeterminate cancers on conventional endoscopy were allocated to ESD, and submucosal cancers were allocated to surgery. After EUS examination, EUS- based treatment plan was modified as follows: m-sm 1 cancers on EUS were allocated to ESD, irrespective of the endoscopy results, and sm2 or advanced cancers on EUS were allocated to surgery. Depth of invasion determined by histologic evaluation was used as the gold standard for the proper selection of treatment modality. Proper selection of treatment was defined that initial treatments by endoscopy or EUS-based treatment plan

Table 1. Patient Characteristics and Clinicopathologic Features of Endoscopically Diagnosed Early Gastric Cancers

\begin{tabular}{|c|c|}
\hline Characteristic & Value \\
\hline \multicolumn{2}{|l|}{ Patients characteristic $(\mathrm{n}=380)$} \\
\hline Age, yr & $62.5 \pm 8.9(38-81)$ \\
\hline Male sex & 297 (78.2) \\
\hline \multicolumn{2}{|l|}{ Tumor characteristic $(n=393)$} \\
\hline Tumor size, $\mathrm{cm}$ & $2.2 \pm 1.2(0.1-8.0)$ \\
\hline$\leq 1.0$ & 78 (19.8) \\
\hline $1.0-2.0$ & $128(32.6)$ \\
\hline $2.0-3.0$ & $116(29.5)$ \\
\hline$>3.0$ & $71(18.1)$ \\
\hline \multicolumn{2}{|l|}{ Macroscopic type } \\
\hline Elevated & $174(44.3)$ \\
\hline Flat & $36(9.2)$ \\
\hline Depressed & $183(46.6)$ \\
\hline \multicolumn{2}{|l|}{ Ulcerative findings } \\
\hline No & 344 (87.5) \\
\hline Yes & $49(12.5)$ \\
\hline \multicolumn{2}{|l|}{ Location within stomach } \\
\hline Lower & 309 (78.6) \\
\hline Middle & $54(13.7)$ \\
\hline Upper & 30 (7.6) \\
\hline \multicolumn{2}{|l|}{ Histology } \\
\hline Papillary & $16(4.1)$ \\
\hline Well differentiated & $266(67.7)$ \\
\hline Moderate differentiated & $111(28.2)$ \\
\hline \multicolumn{2}{|l|}{ Depth of invasion } \\
\hline Mucosa & $271(69.0)$ \\
\hline Submucosa & $102(26.0)$ \\
\hline sm1 & $31(30.4)$ \\
\hline $\mathrm{sm} 2$ & $71(69.6)$ \\
\hline Proper muscle & $20(5.1)$ \\
\hline \multicolumn{2}{|l|}{ Treatment methods } \\
\hline ESD & $168(42.7)$ \\
\hline Additional surgery after ESD & $26(6.6)$ \\
\hline Surgery & 199 (50.6) \\
\hline
\end{tabular}

Data are presented as mean \pm SD (range) or number (\%). $\mathrm{sm} 1$, invasion into the submucosa $<500 \mu \mathrm{m}$ from the muscularis $\mathrm{mu}-$ cosae; sm2, invasion into the submucosa $\geq 500 \mu \mathrm{m}$ from the muscularis mucosae; ESD, endoscopic submucosal dissection. 
coincided with those by histologic evaluation focused on the depth of invasion as follows: mucosal or minute submucosal (sm1) cancers on histologic examination were regarded as appropriate candidates for ESD, whereas deep submucosal (sm2) or advanced cancers (proper muscle or beyond) were regarded as surgical candidates. Unnecessary surgery was defined as initial treatment allocated to surgery by endoscopy or EUS-based treatment plan in mucosal or minute submucosal (sm1) cancers on histologic examination. Additional surgery after ESD was defined that surgical treatment was needed as definite treatment in deep submucosal (sm2) or advanced cancers which were initially allocated to ESD by endoscopy or EUS-based treatment. Proper treatment selection, unnecessary surgery and additional surgery after ESD in this study were not real results of our patients but hypothetical treatment results by simplified hypothetical treatment algorithm focused on the depth of invasion.

\section{Statistical analysis}

Continuous variables are presented as mean \pm standard deviation. Comparisons between endoscopy-based and EUS-based treatment plans for the proper selection of treatment method were analyzed using the McNemar test. Two-sided p-values $<0.05$ were considered significant.

\section{RESULTS}

\section{Clinicopathologic features}

Patient characteristics and clinicopathologic features of the lesions are summarized in Table 1 . The mean age of the patients was 62.5 years (range, 38 to 81 years), and the male:female ratio was 3.6:1 (297:83). The mean tumor diameter was $2.2 \mathrm{~cm}$ (range, 0.1 to $8.0 \mathrm{~cm})$. Of the 393 lesions, $183(46.6 \%)$ were depressed, and $49(12.5 \%)$ exhibited ulcerative findings. The lesions were mainly located in the lower area of the stomach (78.6\%), and 271 (69.0\%) were mucosal cancers on final histologic examination. Of the 194 lesions initially treated by ESD, 26 lesions (6.6\%) were treated with additional surgery because they were incompletely resected or were outside the expanded criteria for ESD. Finally, 225 lesions (57.3\%) were treated by surgery as curative treatment.

\section{Invasion depth assessment by conventional endoscopy and EUS}

Table 2 shows the results of invasion depth, as assessed by conventional endoscopy or EUS. On depth of invasion assessment by endoscopy, the numbers of mucosal, indeterminate, and submucosal cancers were 253 (64.4\%), 56 (14.2\%), and 84 (21.4\%), respectively. On invasion depth assessment by EUS, the numbers of $\mathrm{m}$-sm1, sm2, and advanced cancers were 214 (54.5\%), 166 (42.2\%), and 13 (3.3\%), respectively. Of the cancers classified as indeterminate on conventional endoscopy, final histologic evaluation identified 34 as mucosal or sm1 cancers (60.7\%) and 22 as sm2 or deeper cancers (39.3\%). The overall accuracies of the conventional endoscopy and EUS were 73.6\% (248/337) and 66.7\% (262/393), respectively. Overestimation of EUS (26.5\%) was more frequent than that of conventional endoscopy (13.4\%), whereas underestimation of EUS (6.9\%) was less frequent than that of conventional endoscopy (13.1\%). The sensitivity of EUS for T1m was lower than that of conventional endoscopy, whereas the specificity for T1m was higher than that of conventional endoscopy.

Table 2. Depth of Tumor Invasion, Based on Conventional Endoscopy and Endoscopic Ultrasonography

\begin{tabular}{|c|c|c|c|c|c|c|}
\hline & \multicolumn{3}{|c|}{ Endoscopic depth diagnosis } & \multicolumn{3}{|c|}{ EUS depth diagnosis } \\
\hline & $\begin{array}{l}\text { Mucosa } \\
(\mathrm{n}=253)\end{array}$ & $\begin{array}{l}\text { Indeterminate } \\
\quad(\mathrm{n}=56)\end{array}$ & $\begin{array}{c}\text { Submucosa } \\
(\mathrm{n}=84)\end{array}$ & $\begin{array}{l}\text { Mucosa-sm1 } \\
(\mathrm{n}=214)\end{array}$ & $\begin{array}{l}\text { Submucosa (sm2) } \\
\qquad(\mathrm{n}=166)\end{array}$ & $\begin{array}{l}\text { Proper muscle } \\
\qquad(\mathrm{n}=13)\end{array}$ \\
\hline \multicolumn{7}{|l|}{ Histologic invasion depth } \\
\hline Mucosa-sm1 $(\mathrm{n}=302)$ & 223 & 34 & 45 & 202 & 96 & 4 \\
\hline Submucosa $2(\mathrm{n}=71)$ & 28 & 18 & 25 & 12 & 55 & 4 \\
\hline Proper muscle $(T 2)(n=20)$ & 2 & 4 & 14 & 0 & 15 & 5 \\
\hline Overall accuracy & $248 / 337^{*}$ & \multicolumn{2}{|c|}{$73.6(68.9-78.3)$} & $262 / 393$ & \multicolumn{2}{|c|}{$66.7(62.0-71.3)$} \\
\hline Accuracy $\left(\mathrm{T} 1 \mathrm{~m}^{\dagger}\right)$ & $262 / 337^{*}$ & \multicolumn{2}{|c|}{$77.7(73.3-82.2)$} & $281 / 393$ & \multicolumn{2}{|c|}{$71.5(67.0-76.0)$} \\
\hline Overestimation & $45 / 337^{*}$ & \multicolumn{2}{|c|}{$13.4(9.7-17.0)$} & $104 / 393$ & \multicolumn{2}{|c|}{$26.5(22.1-30.8)$} \\
\hline Underestimation & $44 / 337^{*}$ & \multicolumn{2}{|c|}{$13.1(9.5-16.7)$} & $27 / 393$ & \multicolumn{2}{|c|}{$6.9(4.4-9.4)$} \\
\hline Sensitivity $\left(\mathrm{T}_{1} \mathrm{~m}^{\dagger}\right)$ & $223 / 268$ & \multicolumn{2}{|c|}{$83.2(78.7-87.7)$} & $202 / 302$ & \multicolumn{2}{|c|}{$66.9(61.6-72.2)$} \\
\hline Specificity $\left(\mathrm{T}_{1} \mathrm{~m}^{\dagger}\right)$ & $39 / 69$ & \multicolumn{2}{|c|}{$56.5(44.8-68.2)$} & $79 / 91$ & \multicolumn{2}{|c|}{$86.8(79.9-93.8)$} \\
\hline PPV $\left(\mathrm{T}_{1} \mathrm{~m}^{\dagger}\right)$ & $223 / 253$ & \multicolumn{2}{|c|}{$88.1(84.2-92.1)$} & $202 / 214$ & \multicolumn{2}{|c|}{$94.4(91.3-97.5)$} \\
\hline NPV $\left(\mathrm{T}_{1} \mathrm{~m}^{\dagger}\right)$ & $39 / 84$ & \multicolumn{2}{|c|}{$46.4(35.8-57.1)$} & $79 / 179$ & \multicolumn{2}{|c|}{$44.1(36.9-51.4)$} \\
\hline
\end{tabular}

Data are presented as number or \% (95\% confidence interval).

EUS, endoscopic ultrasonography; PPV, positive predictive value; NPV, negative predictive value.

*Excludes 56 indeterminate cancers for calculation; ${ }^{\dagger} \mathrm{T} 1 \mathrm{~m}$ represents mucosal or minute submucosal (sm1) cancer on endoscopic and EUS-based depth evaluations. 


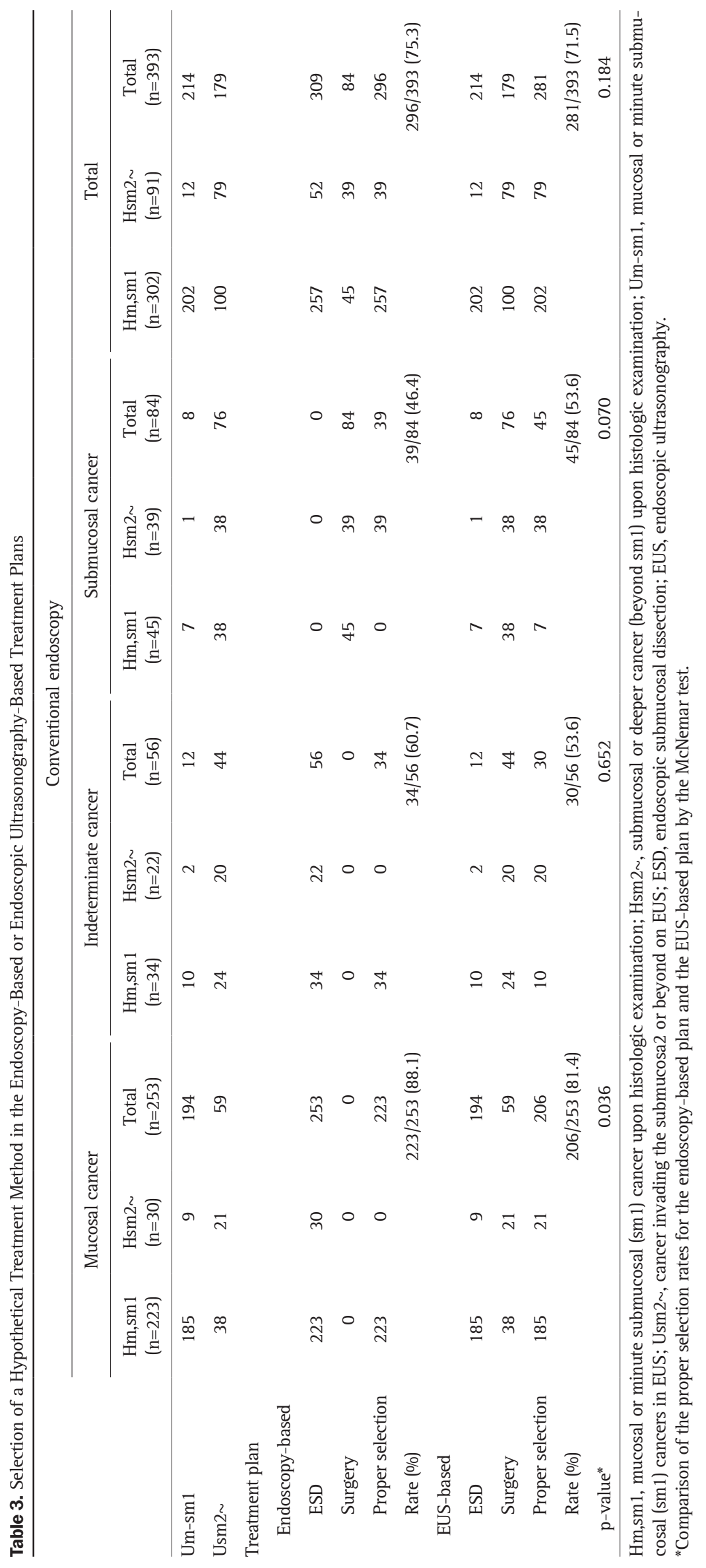




\section{Proper selection of treatment method by endoscopy- based or EUS-based treatment plan}

Results for the selection of hypothetical treatment method according to endoscopy-based or EUS-based plan by simplified hypothetical treatment algorithm are summarized in Table 3 and Fig. 2. Mucosal or indeterminate cancers on conventional endoscopy were allocated to ESD, and submucosal cancers were allocated to surgery. Proper treatment selection rates by endoscopy-based plan were 88.1\% (223/253) for mucosal cancers, 60.7\% (34/56) for indeterminate cancers, 46.4\% (39/84) for submucosal cancers, and 75.3\% (296/393) for all tumors. The percentages of proper selection by EUS-based plan were 81.4\% (206/253) for endoscopic mucosal cancers, 53.6\% (30/56) for indeterminate cancers, 53.6\% (45/84) for submucosal cancers, and 71.5\% (281/393) for all tumors. For endoscopic mucosal cancers, EUS-based plan significantly reduced the proper selection rate $(\mathrm{p}=0.036)$. Proper treatment selection rates did not differ between the two methods for endoscopic indeterminate cancers ( $p=0.652$ ) and endoscopic submucosal cancers ( $p=0.070)$. For EGCs overall, the proper treatment selection rates did not differ between endoscopy-based and EUS-based treatment plans $(\mathrm{p}=0.184)$.

For endoscopy-based treatment plan, surgery was necessary in 52 EGCs (13.2\%) after ESD, and unnecessary surgery was performed in 45 cancers (11.5\%). EUS-based plan changed the therapeutic strategies in 28.2\% (111/393) of EGCs: ESD was changed to surgery in 23.3\% (59/253) of endoscopic mucosal cancers and 78.6\% (44/56) of indeterminate cancers, and surgery was changed to ESD in 9.5\% (8/84) of endoscopic submucosal cancers. Surgery was required in 12 EGCs (3.1\%) after ESD and unnecessary surgery was performed in 100 cases (25.4\%).

\section{DISCUSSION}

In the preoperative staging of EGC, the prediction of tumor invasion depth is essential for therapeutic decision-making. Thus, we compared the effectiveness of EUS-based treatment plan with that of endoscopy-based treatment plan for properly selecting the EGC treatment method focused on the prediction of invasion depth. Our data showed that EUS-based plan did not increase the rate of proper selection of treatment method in EGCs, and it even decreased the rate in the subgroup of EGCs identified as mucosal cancers by endoscopy.

Several studies have reported that $15 \%$ to $26 \%$ of patients treated by endoscopic resection have required additional treatment, including surgical resection, because of noncurative resection. ${ }^{1,19-22}$ A considerable number of these additional surgical treatments were required for massive submucosal invasion (beyond sm1) (19\% to 44\%). ${ }^{1,19-21}$ Although EUS has been regarded as the best method for the locoregional assessment of gastric cancer (with a high accuracy), ${ }^{6-8}$ controversy remains whether EUS is efficacious in being able to distinguish between mucosal and deeper tumors, and thus reduce the rate of inappropriate treatment selection. Yanai et al. ${ }^{12}$ reported that EUS is useful for the assessment of invasion depth, especially in instances when the depth is underestimated by conventional endoscopy. In addition, Okada et al. ${ }^{9}$ reported that EGCs meeting the expanded indication criteria for ESD can be diagnosed with a high accuracy by EUS. On the other hand, other studies reported that differentiated mucosal EGCs $<2 \mathrm{~cm}$ were precisely staged using endoscopy alone, ${ }^{5}$ and the use of EUS did not increase the accuracy for invasion depth assessment of EGC over the accuracy associated with the use of conventional endoscopy alone. ${ }^{10,11}$ These discrepancies may be due to differences between the studies in study populations (all EGCs vs difficult cases by endoscopy), EUS instrument (miniprobe only vs radial echoencoscope

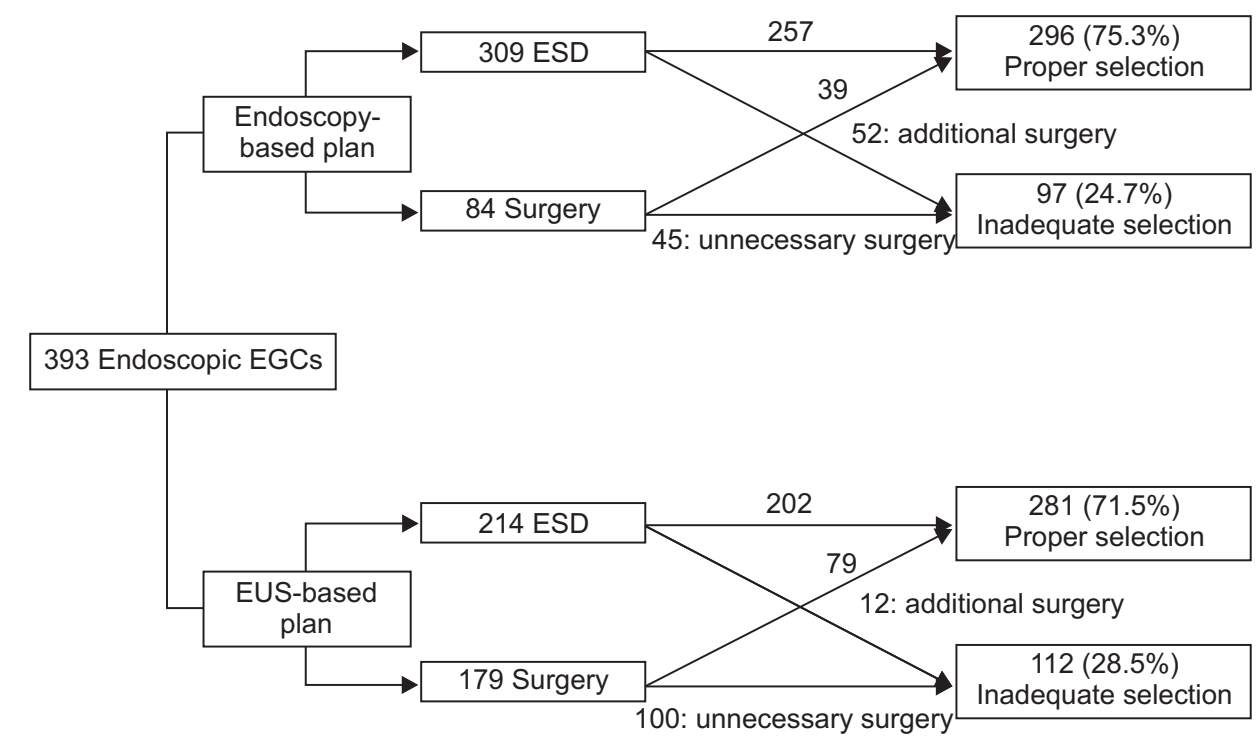

Fig. 2. Flowchart of hypothetical treatment selection in the endoscopy-based or the endoscopic ultrasonography (EUS)-based plans. This figure shows the results of hypothetical treatment selection according to endoscopy-based or EUS-based plans, generated by a simplified hypothetical treatment algorithm. EGC, early gastric cancer; ESD, endoscopic submucosal dissection. 
or miniprobe), EUS frequencies (12 $\mathrm{MHz}$ vs $20 \mathrm{MHz}$ ), and EUS invasion depth assessment (blind assessment using photoprints vs retrospective review of medical records).

Endoscopic assessment of invasion depth (mucosa vs submucosa) of EGC is based on the tumor's surface nodularity, stiffness, and fold morphology, and the accuracy of endoscopic assessment is affected by tumor size, gross morphology, ulceration, location, histologic differentiation, and depth of invasion. ${ }^{3,412}$ However, there are no standard endoscopic criteria for differentiating mucosal cancers from deeper tumors. Furthermore, some EGCs have ambiguous morphology, which leads to difficulties in evaluating the depth of invasion. In our study, we examined whether EUS is specifically helpful for these tumors. In our hypothetical treatment algorithms, indeterminate cancers on conventional endoscopy were allocated to ESD because less invasive treatment was favored for these lesions. We found that the accuracy rate of EUS for indeterminate cancers was 53.6\% (30/56), and the proper selection rates for endoscopy-based plan (60.7\%) and EUS-based plan (53.6\%) were similar ( $p=0.652)$. Moreover, EUS-based plan resulted in a considerable number of unnecessary surgeries in this group (24/56, 42.9\%). Thus, EUS did not improve the proper treatment selection rates in EGCs with ambiguous morphology on endoscopy.

Our results also showed that EUS-based plan did not improve the proper selection of treatment method in EGCs overall, as the rate of proper treatment selection was 75.3\% with endoscopy and $71.5 \%(p=0.184)$ with EUS. This failure of EUS to improve the proper selection rate may be due to one or more of the following explanations. First, conventional endoscopy alone has a sufficient diagnostic accuracy in predicting tumor depth in EGC. One large-scale study reported that conventional endoscopy alone had a diagnostic accuracy of 78\%, and a positive predictive value for mucosal cancers of $82.0 \%$, thereby suggesting that endoscopy is sufficient for predicting tumor depth in EGC, without the need for EUS. ${ }^{4}$ Second, the diagnostic accuracy for distinguishing mucosal cancers from submucosal cancers was not significantly different between conventional endoscopy and EUS. According to previous studies, the accuracy rates of conventional endoscopy $(72 \% \text { to } 84 \%)^{3-5}$ did not differ from those of EUS (67\% to $82 \%) .{ }^{5,9,10}$ Third, the tendency of EUS to overestimate the depth of invasion might lower the proper selection rate. This overestimation tendency of EUS has been reported in previous studies. ${ }^{10,12,23}$ In our study, the overestimation rate (26.5\%) of EUS was higher than that of conventional endoscopy (13.4\%). Overestimation during EUS occurs with ulceration or fibrosis in the cancer, which is difficult to distinguish from submucosal tumor invasion, whereas endoscopy can differentiate between cancer invasion and ulcer fibrosis more easily than EUS. ${ }^{5,10}$ Taking the aforementioned into consideration, EUS may not increase the diagnostic accuracy of tumor depth estimation. Furthermore, the overestimation tendency of EUS might increase the number of unnecessary surgeries. Our results demon- strated that the rate of unnecessary surgeries as initial treatment was greatly increased (11.5\% to 25.4\%) by EUS-based plan.

EGCs exhibiting a differentiated histology type with minute submucosal penetration ( $\mathrm{sm} 1[<500 \mu \mathrm{m}$ from the muscularis mucosae]), no lymphatic-vessel invasion, and size $\leq 3 \mathrm{~cm}$ are thought to have a nominal risk of lymph node metastasis and have been included in the expanded indications for ESD. ${ }^{24}$ However, during the preoperative evaluation, it is quite difficult to differentiate sm 1 cancers from mucosal cancers because minute submucosal invasive lesions rarely exhibit morphological characteristics on conventional endoscopy, and because they are also barely detectable on EUS secondary to limitations of EUS resolution. ${ }^{25-27}$ Despite these limitations of conventional endoscopy and EUS in differentiating sm 1 from mucosal cancers, most sm 1 cancers $<3 \mathrm{~cm}$ are within the expanded indications of ESD, and the treatment plan for these lesions is the same as for mucosal cancers. ${ }^{24,28}$ Therefore, we regarded ESD as the proper therapeutic plan for lesions with invasion within the mucosal or sm1 layers on EUS.

A major advantage of our study was our use of prospectively collected conventional endoscopy and EUS data, with real-time diagnosis. In previous studies, endoscopic evaluation and EUS assessment of tumor invasion were performed by retrospective review of recorded still images. ${ }^{4,5,10-12}$ However, it is difficult to detect the stiffness of the EGC base by reviewing images. Furthermore, minute submucosal invasion on EUS is prone to remain undetected if images are not recorded accurately, and oblique scanned images tend to overestimate the depth of invasion. Thus, our real-time evaluation of endoscopy and EUS may have provided a particularly accurate evaluation of the two techniques. In addition, our study population was not limited to EGCs with indications for ESD. All EGCs with differentiated histology type were collected to reduce selection bias and to evaluate the effectiveness of EUS-based plan to the actual diagnostic process.

Our study has limitations. First, EUS examinations were performed using a radial echoendoscope. Some authors have reported that the accuracy of the high frequency miniprobe is higher than that of radial EUS. ${ }^{10,29,30}$ Thus, the use of a radial echoendoscope might have influenced the diagnostic accuracy of EUS in our study. However, the radial echoendoscope (GF-UM2000; Olympus) we used is switchable between four frequencies: 5, 7.5, 12, and $20 \mathrm{MHz}$. This frequency range is excellent for precise invasion depth assessment, as well as lymph node assessment. Secondly, EUS evaluation was performed by endoscopists who were not blinded to the results of the conventional endoscopy. Thus, the interpretation of EUS might have been affected by the results of the conventional endoscopy. However, performing EUS while being blinded to the results of conventional endoscopy is difficult to accomplish during the actual diagnostic process of EGC. Thirdly, majority of our study populations were mucosal cancers on final histologic examina- 
tion $(271 / 393,69.0 \%)$. Thus, this proportion might cause the selection bias of favoring conventional endoscopy. Fourthly, only differentiated-type EGCs were included in the analysis. Thus, this point should be taken into consideration in applying our results.

In conclusion, our study showed that EUS examination did not increase the proper selection of treatment in differentiatedtype EGCs, and it increased the rate of unnecessary surgery in selected cases. Thus, routine EUS examination may not be necessary in the preoperative assessment of differentiated-type EGC, especially for endoscopically-presumed mucosal cancers before ESD.

\section{CONFLICTS OF INTEREST}

No potential conflict of interest relevant to this article was reported.

\section{ACKNOWLEDGEMENTS}

This work was supported by Grant 1310280 from the National Cancer Center, Korea.

\section{REFERENCES}

1. Isomoto H, Shikuwa S, Yamaguchi N, et al. Endoscopic submucosal dissection for early gastric cancer: a large-scale feasibility study. Gut 2009;58:331-336.

2. Chung IK, Lee JH, Lee SH, et al. Therapeutic outcomes in 1000 cases of endoscopic submucosal dissection for early gastric neoplasms: Korean ESD Study Group multicenter study. Gastrointest Endosc 2009;69:1228-1235.

3. Sano T, Okuyama Y, Kobori O, Shimizu T, Morioka Y. Early gastric cancer: endoscopic diagnosis of depth of invasion. Dig Dis Sci 1990;35:1340-1344.

4. Choi J, Kim SG, Im JP, Kim JS, Jung HC, Song IS. Endoscopic prediction of tumor invasion depth in early gastric cancer. Gastrointest Endosc 2011;73:917-927.

5. Hizawa K, Iwai K, Esaki M, Matsumoto T, Suekane H, Iida M. Is endoscopic ultrasonography indispensable in assessing the appropriateness of endoscopic resection for gastric cancer? Endoscopy 2002;34:973-978.

6. Polkowski M, Palucki J, Wronska E, Szawlowski A, NasierowskaGuttmejer A, Butruk E. Endosonography versus helical computed tomography for locoregional staging of gastric cancer. Endoscopy 2004;36:617-623.

7. Kwee RM, Kwee TC. Imaging in local staging of gastric cancer: a systematic review. J Clin Oncol 2007;25:2107-2116.

8. Mocellin S, Marchet A, Nitti D. EUS for the staging of gastric cancer: a meta-analysis. Gastrointest Endosc 2011;73:1122-1134.

9. Okada K, Fujisaki J, Kasuga A, et al. Endoscopic ultrasonography is valuable for identifying early gastric cancers meeting expanded- indication criteria for endoscopic submucosal dissection. Surg Endosc 2011;25:841-848.

10. Choi J, Kim SG, Im JP, Kim JS, Jung HC, Song IS. Comparison of endoscopic ultrasonography and conventional endoscopy for prediction of depth of tumor invasion in early gastric cancer. Endoscopy 2010;42:705-713.

11. Choi J, Kim SG, Im JP, Kim JS, Jung HC, Song IS. Is endoscopic ultrasonography indispensable in patients with early gastric cancer prior to endoscopic resection? Surg Endosc 2010;24:3177-3185.

12. Yanai H, Noguchi T, Mizumachi S, et al. A blind comparison of the effectiveness of endoscopic ultrasonography and endoscopy in staging early gastric cancer. Gut 1999;44:361-365.

13. The Paris endoscopic classification of superficial neoplastic lesions: esophagus, stomach, and colon: November 30 to December 1, 2002. Gastrointest Endosc 2003;58(6 Suppl):S3-S43.

14. Aibe T, Fuji T, Okita K, Takemoto T. A fundamental study of normal layer structure of the gastrointestinal wall visualized by endoscopic ultrasonography. Scand J Gastroenterol Suppl 1986;123:615.

15. Kimmey MB, Martin RW, Haggitt RC, Wang KY, Franklin DW, Silverstein FE. Histologic correlates of gastrointestinal ultrasound images. Gastroenterology 1989;96(2 Pt 1):433-441.

16. Kida M, Tanabe S, Watanabe M, et al. Staging of gastric cancer with endoscopic ultrasonography and endoscopic mucosal resection. Endoscopy 1998;30 Suppl 1:A64-A68.

17. Hamilton SR, Aaltonen LA; World Health Organization; International Agency for Research on Cancer. Pathology and genetics of tumours of the digestive system. Lyon: IARC Press, 2000:37-52.

18. Japanese Gastric Cancer Association. Japanese classification of gastric carcinoma: 2nd English edition. Gastric Cancer 1998;1:1024.

19. Oda I, Gotoda T, Sasako M, et al. Treatment strategy after noncurative endoscopic resection of early gastric cancer. Br J Surg 2008;95:1495-1500.

20. Ryu KW, Choi IJ, Doh YW, et al. Surgical indication for non-curative endoscopic resection in early gastric cancer. Ann Surg Oncol 2007;14:3428-3434.

21. Choi MK, Kim GH, Park do Y, et al. Long-term outcomes of endoscopic submucosal dissection for early gastric cancer: a singlecenter experience. Surg Endosc 2013;27:4250-4258.

22. Ahn JY, Jung HY, Choi KD, et al. Endoscopic and oncologic outcomes after endoscopic resection for early gastric cancer: 1370 cases of absolute and extended indications. Gastrointest Endosc 2011;74:485-493.

23. Kim JH, Song KS, Youn YH, et al. Clinicopathologic factors influence accurate endosonographic assessment for early gastric cancer. Gastrointest Endosc 2007;66:901-908.

24. Soetikno R, Kaltenbach T, Yeh R, Gotoda T. Endoscopic mucosal resection for early cancers of the upper gastrointestinal tract. J Clin Oncol 2005;23:4490-4498.

25. Sabet EA, Okai T, Minamoto T, Mai M, Sawabu N. Visualizing the gastric wall with a 30-MHz ultrasonic miniprobe: ex vivo imaging 
50 Gut and Liver, Vol. 10, No. 1, January 2016

of normal gastric sites and sites of early gastric cancer. Abdom Imaging 2003;28:252-256.

26. Mouri R, Yoshida S, Tanaka S, Oka S, Yoshihara M, Chayama $\mathrm{K}$. Usefulness of endoscopic ultrasonography in determining the depth of invasion and indication for endoscopic treatment of early gastric cancer. J Clin Gastroenterol 2009;43:318-322.

27. Matsumoto Y, Yanai H, Tokiyama H, Nishiaki M, Higaki S, Okita K. Endoscopic ultrasonography for diagnosis of submucosal invasion in early gastric cancer. J Gastroenterol 2000;35:326-331.
28. Japanese Gastric Cancer Association. Japanese gastric cancer treatment guidelines 2010 (ver. 3). Gastric Cancer 2011;14:113-123.

29. Akahoshi K, Chijiwa Y, Hamada S, et al. Pretreatment staging of endoscopically early gastric cancer with a $15 \mathrm{MHz}$ ultrasound catheter probe. Gastrointest Endosc 1998;48:470-476.

30. Hunerbein M, Handke T, Ulmer C, Schlag PM. Impact of miniprobe ultrasonography on planning of minimally invasive surgery for gastric and colonic tumors. Surg Endosc 2004;18:601-605. 